\title{
Tensile fracture during transformation superplasticity of Ti-6Al-4V
}

\author{
C. Schuh and D.C. Dunand \\ Department of Materials Science and Engineering, Northwestern University, \\ 2225 North Campus Drive, Room 2036, Evanston, Illinois 60208
}

(Received 6 September 2000; accepted 4 January 2001)

\begin{abstract}
During thermal cycling through the $\alpha-\beta$ phase transformation under the action of a small external biasing stress, Ti alloys exhibit an average deformation stress exponent of unity and achieve superplastic strains. We report tensile experiments on Ti-6Al-4V with an applied stress of $4.5 \mathrm{MPa}$, aimed at understanding the failure processes during transformation superplasticity. The development of cavities was assessed as a function of superplastic elongation, and macroscopic neck formation was quantified at several levels of elongation by digital imaging techniques. The effects of thermal inhomogeneity on neck initiation and propagation were also elucidated experimentally. Tensile ductility during transformation superplasticity is compared with that during isothermal creep at the average, effective cycling temperature, and a numerical model is used to show the effect of thermal gradients in limiting superplastic elongation.
\end{abstract}

\section{INTRODUCTION}

Transformation superplasticity (TSP) is a deformation mechanism observed in polymorphic materials subjected to cyclic phase transformation under an externally applied stress. ${ }^{1}$ Internal transformation mismatch strains are biased in the direction of the external stress, resulting in deformation with a low stress sensitivity. In this manner, repeated transformations (by, e.g., thermal cycling) can produce superplastic strains. With the demonstration of TSP in advanced metallic materials (i.e., intermetallic alloys ${ }^{2,3}$ and metal-matrix composites), ${ }^{3-7}$ as well as the extension of this mechanism to multiaxial modes of deformation, ${ }^{8}$ TSP is of current interest as a prospective shape-forming technology.

Studies of TSP to date have focused primarily on the mechanics of steady-state deformation, with some limited studies on post-deformation mechanical properties. $^{6,9,10}$ In addition, failure during internal-stress superplasticity, in which internal stresses are caused by thermal-expansion mismatch between coexisting phases, has been examined in an $\mathrm{Al}-\mathrm{SiC}$ composite in Ref. 11. However, to our knowledge there have been no systematic studies describing the mechanisms of failure during TSP, in uniaxial tension or otherwise. For implementation of TSP in shape-forming processes, knowledge of damage evolution, including cavitation and macroscopic plastic instability development, is essential for process design.

The goal of the present work is to identify the governing process parameters that influence tensile fracture during TSP of Ti-6Al-4V. Internal cavity volume frac- tion and macroscopic shape instabilities are examined as a function of superplastic strain. Experiments that elucidate the effect of thermal variations on plastic instability are described, and TSP fracture is compared with isothermal creep fracture at the average, or effective, thermal cycling temperature.

\section{EXPERIMENTAL PROCEDURES}

\section{A. General procedures}

Powder metallurgical Ti-6Al-4V was supplied by Dynamet Technology (Burlington, MA), produced by cold pressing of blended elemental powders, sintering, and hot-isostatic pressing, in a process described in Ref. 12. The microstructure of the material exhibits large colonies of $\alpha$ plates, as shown in Fig. 1. The as-received material was machined into cylindrical tensile specimens with a $20-\mathrm{mm}$ gauge length of $5-\mathrm{mm}$ diameter. The fillet radius between the specimen gauge and head was sharp (about $0.5 \mathrm{~mm}$ ). The specimens were deformed in tension in a custom creep frame described in detail elsewhere, ${ }^{13}$ under an atmosphere of purified argon. The specimens were heated by four radiant line heaters, positioned symmetrically around the load train and focused onto the tension axis.

To establish the constitutive isothermal mechanical behavior of Ti-6Al-4V at low stresses and high temperatures of interest to the present work, several creep experiments were conducted at a constant temperature of $970{ }^{\circ} \mathrm{C}$ (in the two-phase $\alpha+\beta$ field) at low stresses. 


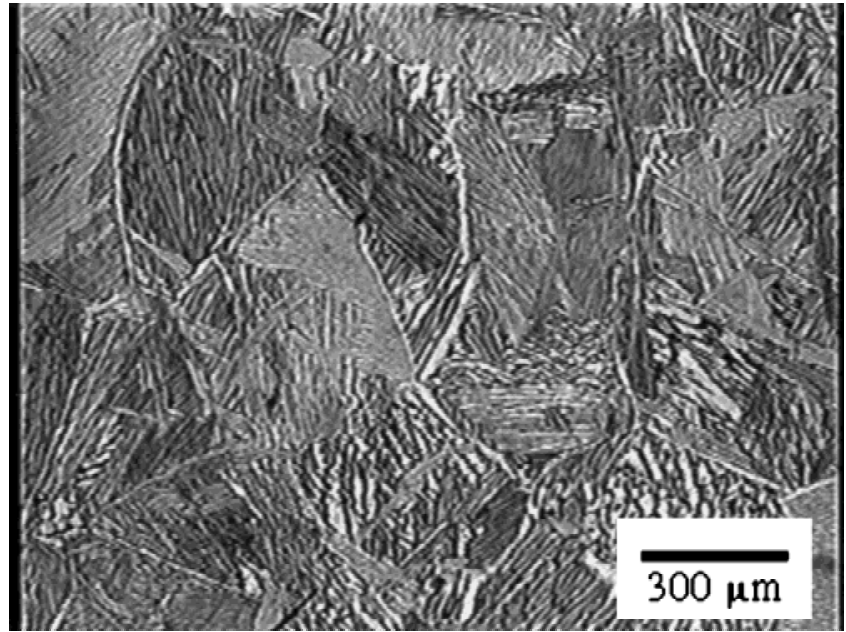

FIG. 1. Optical micrograph of as-received Ti-6Al-4V material used in the present work, etched with Kroll's reagent.

Three types of tensile fracture tests under isothermal and thermal cycling conditions (which are described in Secs. II. B-II. D) were performed, under a nominal uniaxial true tensile stress of $\sigma_{\mathrm{n}}=4.5 \pm 0.2 \mathrm{MPa}$, computed by assuming uniform gauge deformation and volume constancy. The development of internal cavities and external necks was investigated during three separate experiments, in which the specimen was deformed to successive strains interspersed with excursions to room temperature. During the excursions to room temperature, the following two measurements were made: (i) Specimen density was determined by using the Archimedes method in distilled water. The water temperature was measured with an accuracy of $0.1 \mathrm{~K}$, and the water density was corrected for temperature according to Ref. 14 . The density of the gauge section was calculated by assuming that the specimen heads remained at full theoretical density for all stages of deformation. After the specimen had failed, the heads were removed from the gauge with a diamond saw, and their density was measured. This procedure indeed verified that the specimen heads remained at full theoretical density. This technique was found to give precise gauge densities to $\pm 3 \cdot 10^{-3} \mathrm{~g} / \mathrm{cm}^{3}( \pm 0.07 \%$ for Ti-6Al-4V). (ii) The macroscopic profile of the gauge section was assessed by the following methods. A digital image of the specimen was first captured, and an edge-finding algorithm was used to obtain the profile of the gauge section. The projected diameter was then measured as a function of axial position using image-processing software. Two perpendicular projections of the specimen were analyzed after each stage of straining. This method gave accurate measurements, with precision of about $60 \mu \mathrm{m}$ on the diameter measurements. In addition, calipers (with accuracy of
$10 \mu \mathrm{m})$ were used to measure the specimen diameter at 6-12 axial positions, to verify the accuracy of the image analysis results.

\section{B. Thermal shielding experiments during TSP}

The first experiment was conducted to examine effects of thermal shielding on TSP deformation. The $\alpha-\beta$ transformation was produced by performing triangular thermal cycles between 840 and $1030{ }^{\circ} \mathrm{C}$ with an 8 -min period. These thermal cycles were chosen to maximize the volume of transformation product (approximately $80 \%{ }^{15}$ ) while providing ample time for complete diffusive transformation. ${ }^{15}$ Temperature was controlled by a $\mathrm{BN}$-coated type-K thermocouple (1.6-mm diameter) positioned at the gauge surface, approximately $7 \mathrm{~mm}$ from the upper specimen head. This arrangement provided a good measurement of the gauge temperature, but it also caused some shadowing of the upper portion of the gauge from the incident radiation. A second thermocouple was positioned at the top of the upper specimen head and did not induce any shadowing on the gauge section.

Before the first stage of deformation, the specimen and load train were allowed to come to thermal equilibrium at the upper cycling temperature $\left(1030^{\circ} \mathrm{C}\right)$ for $75 \mathrm{~min}$, after which the load was applied and thermal cycling was initiated. This procedure was used to ensure a coarse $\beta$-grain size (approximately $0.5-1 \mathrm{~mm}$ ), thereby minimizing subsequent grain growth during thermal cycling. After this treatment, at room temperature, a colony structure like that shown in Fig. 1 is still observed, but with larger prior $\beta$-grains. A similar equilibration procedure was performed before each subsequent stage of deformation, but at the lower cycling temperature $\left(840{ }^{\circ} \mathrm{C}\right)$ to minimize thermal sintering of any internal cavities. The deformation was interrupted six times; together with data collected before deformation and after fracture, this allowed for density and profile measurements at eight values of gauge elongation between $0 \%$ and $260 \%$.

In addition to the above experiments, heat transfer effects were considered during the third stage of deformation (from 32\% to 66\% elongation), when the second sheathed type-K thermocouple (1.6-mm diameter) was extended from its original position at the upper specimen head to the lower head of the specimen. This thermocouple acted as a radiation barrier, blocking an estimated $10-20 \%$ of the incident radiation from the heaters, across the full gauge length of the specimen.

\section{Unshielded TSP experiment}

To reduce thermal variations imposed by the positions of thermocouples (as examined explicitly in the previous experiment), the following second TSP experiment was conducted, again following a 75 -min anneal at $1030{ }^{\circ} \mathrm{C}$ before the first application of stress. At the beginning of 
each stage of deformation, the specimen was thermally cycled five times (again over the range $840-1030{ }^{\circ} \mathrm{C}$ in triangular cycles of 8-min duration) without applied stress, during which time both the head and gauge temperatures were monitored. The gauge temperature was used to calibrate the head temperature, and the gauge thermocouple was then removed from the hot zone. The calibrated head thermocouple was then used to control the heaters to produce accurate thermal cycles of the gauge section, but without causing any thermal shielding due to thermocouple positioning. This thermal calibration procedure was repeated at each stage of deformation, and the calibration varied only slightly over the course of the experiment.

\section{Isothermal creep experiment}

For comparison with the two TSP experiments, a third specimen was crept isothermally to failure, and the density and specimen profiles were monitored after several stages of deformation. As with the TSP experiments, this specimen was first annealed at $1030{ }^{\circ} \mathrm{C}$ for 75 min before the first application of stress. The creep temperature was equal to the so-called effective temperature, $T_{\text {eff }}$, of the thermal cycling experiments. At this temperature, the isothermal creep rate is that expected on average during thermal cycling, in the absence of an enhancement due to the phase transformation. The effective temperature is discussed in Refs. 4 and 16 for thermal cycles involving thermal-expansion mismatch of a composite and an allotropic phase transformation, respectively. In the case of Ti-6Al-4V, the phase transformation occurs over most of the thermal cycle, so the calculation of $T_{\text {eff }}$ is more complicated. The calculation of the effective temperature of the thermal cycles used here is presented in the Appendix by using experimental data on the isothermal creep of Ti-6Al-4V, giving $T_{\text {eff }}=982{ }^{\circ} \mathrm{C}$. The isothermal creep fracture experiment was conducted at this temperature, for which the equilibrium volume fraction of $\beta$-phase is $f_{\beta}=0.86 .{ }^{15}$ The thermal calibration method described in Sec. II. C was used, in which the calibrated head temperature was used to control the heaters. Thus, no thermocouple probes or other obstructions caused radiation shielding of the specimen gauge.

\section{RESULTS}

\section{A. Stress dependence of deformation and cavitation during isothermal creep and TSP}

Figure 2 shows the strain rates $\dot{\epsilon}$ measured during isothermal creep of two different specimens at $970{ }^{\circ} \mathrm{C}$, as a function of the applied tensile stress $\sigma$. A power law with stress exponent of approximately 2.1 is observed. For comparison, the average strain rate during thermal cycling $\left(840-1030{ }^{\circ} \mathrm{C}\right.$, triangular, 8-min cycles) is also shown, from our previous work on similar material. ${ }^{9}$ TSP is characterized by higher strain rates than creep at the effective temperature, and by a low stress exponent near unity, as previously discussed in Ref. 17.

The volume fraction of cavities measured after each stage of deformation is shown in Fig. 3 for the three interrupted tensile fracture experiments. The amount of

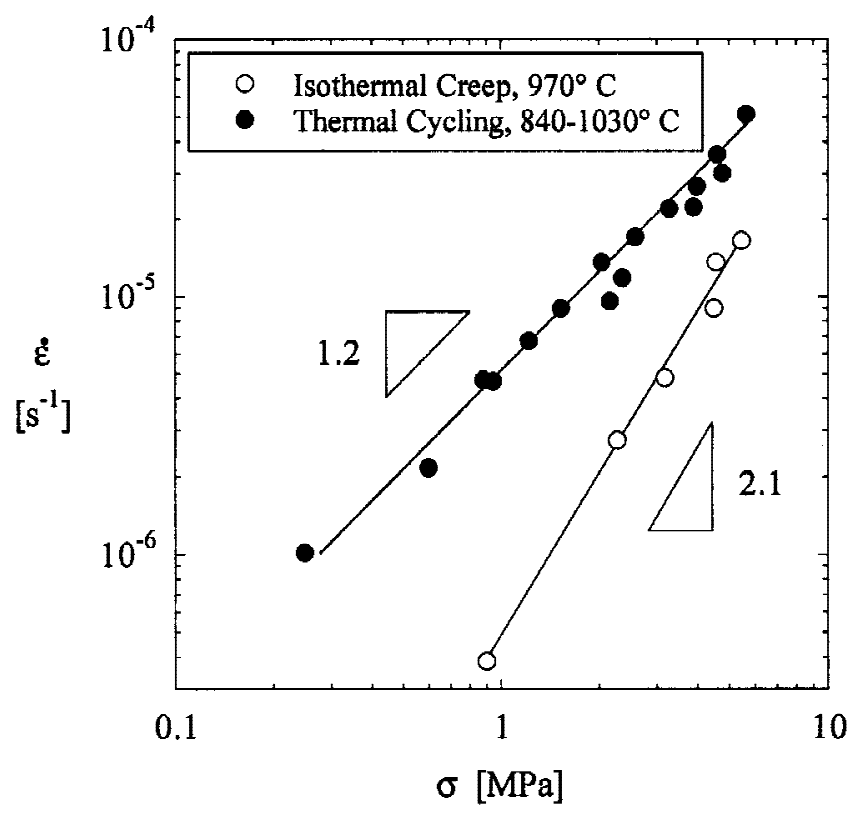

FIG. 2. Stress dependence of average creep rate under isothermal conditions (present study) and thermal cycling, TSP conditions (Ref. 9) for Ti-6Al-4V.

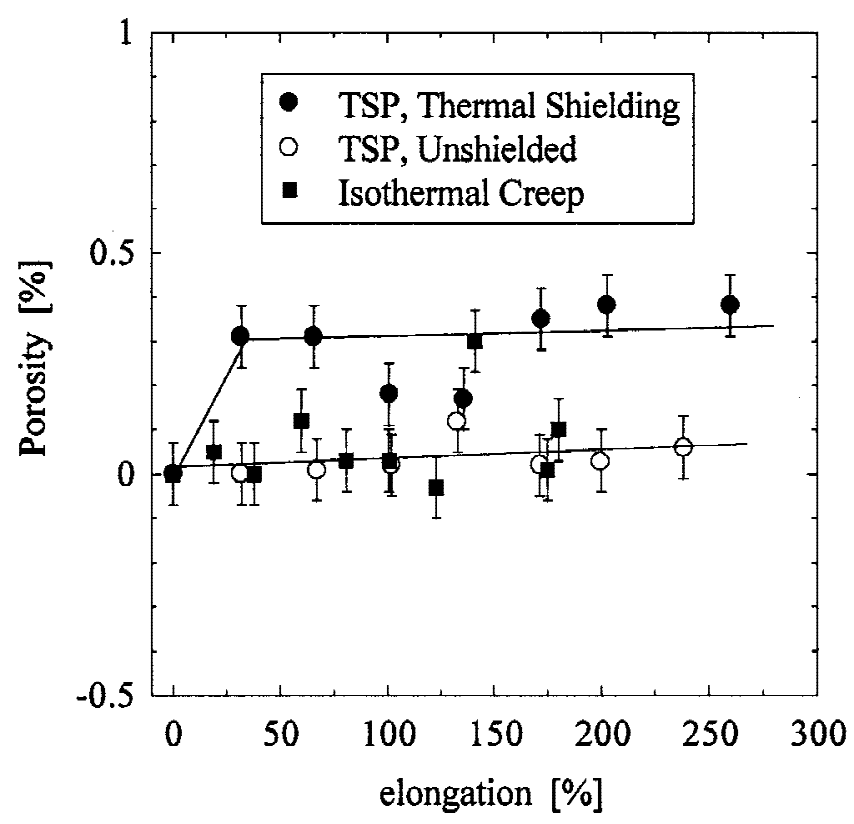

FIG. 3. Porosity of the gauge section of Ti-6Al-4V as a function of elongation (engineering strain). 
deformation-induced cavitation was $<0.4 \%$ for all stages of deformation, for both TSP experiments and the isothermal creep experiment.

\section{B. Tensile fracture experiments}

After all stages of straining, for all three test conditions, the specimens exhibited circular cross sections, with only slight deviations from circular symmetry (approximately 5\%). Thus, the two orthogonal digital images yielded specimen profiles that overlapped to within $20 \mu \mathrm{m}$ in most cases and to within $70 \mu \mathrm{m}$ in the worst case. In addition, the diameter caliper measurements agreed well with the data collected from digital image processing. Therefore, the diametral measurements from the two images were averaged to determine the specimen profiles.

\section{Thermal shielding effects on TSP deformation}

The measured displacements during thermal cycling are composed of thermal expansion and contraction of the specimen and load train, as well as deformation of the specimen by TSP and creep. Thermal expansion displacements are fully recovered after a complete thermal cycle, allowing for the measurement of a true strain increment, $\Delta \epsilon$, after each thermal cycle, which represents deformation of the specimen, averaged over the gauge length. In Fig. 4, the full deformation history of the thermal-shielding experiment is shown, in which each strain increment $\Delta \epsilon$ is normalized by the nominal applied stress, $\sigma_{\mathrm{n}}$, and plotted against the cycle number, $N$. Ver-

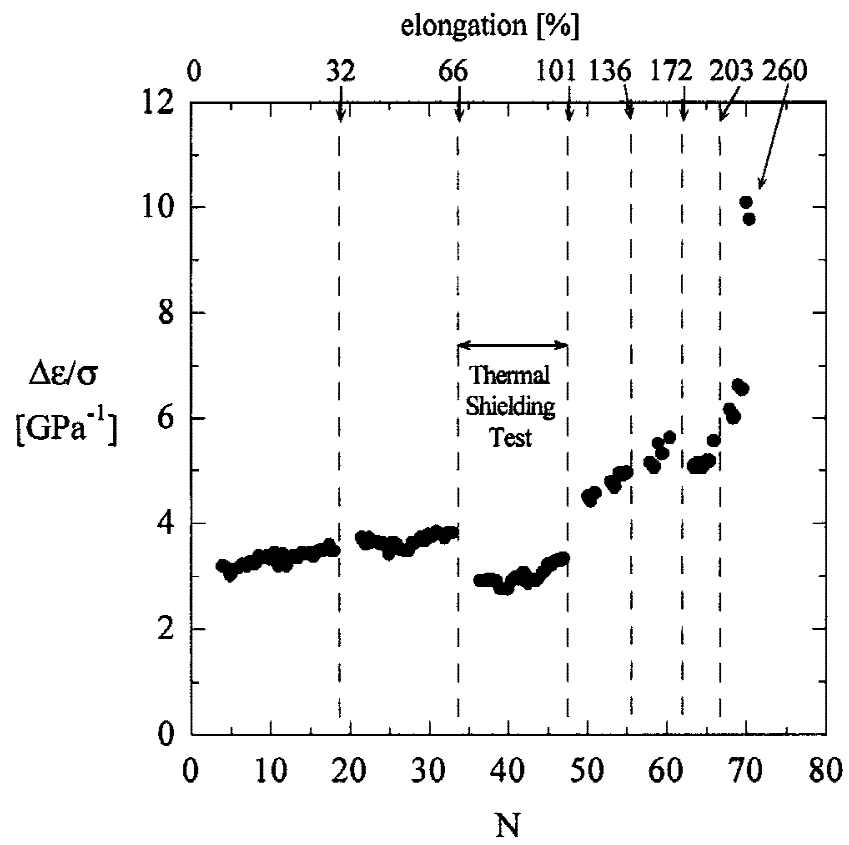

FIG. 4. Deformation history for the thermal shielding experiment, showing the stress-normalized strain increment $\Delta \epsilon / \sigma$ as a function of thermal cycle number $N$. tical dotted lines are shown where the test was interrupted. At the beginning of each stage of deformation, the measured displacement is complicated by thermal transients, which disappear after a dynamic steady-state is established (typically requiring about 2-3 thermal cycles); data points associated with these transient cycles have been removed from Fig. 4.

As seen in Fig. 4, the normalized strain increment, $\Delta \epsilon / \sigma_{\mathrm{n}}$, was approximately $3.1 \mathrm{GPa}^{-1}$ at the beginning of the test, rising to larger values over the course of the experiment. For the stage of deformation in which thermal shielding was investigated, $\Delta \epsilon / \sigma_{n}$ was reduced by about $25 \%$ relative to the data points of the immediately preceding and following stages.

Finally, Fig. 5 shows the gauge section profiles after each stage of deformation, including the measured diameter and the cross-sectional area (calculated from the average diameter). Beginning at the early stages of the test, the specimen exhibited a tendency to deform homogeneously, except near the upper head (left side of Fig. 5), where deformation proceeded more slowly. This inhomogeneity resulted in a neck (arrows in Fig. 5) that ultimately thinned until the specimen failed.

\section{Fracture during creep and TSP}

Fig. 6 compares the deformation histories measured during the unshielded creep and TSP experiments. The average thermal-cycling creep rate is calculated by dividing $\Delta \epsilon$ by the cycle duration. This figure highlights two advantages of TSP over isothermal creep for prospective use in shape-forming applications. First, TSP exhibits faster deformation rates at low applied stresses (4.5 MPa in the present case); at the outset of straining in Fig. 6, the TSP strain rate is about three times higher than for creep at the effective temperature of the thermal

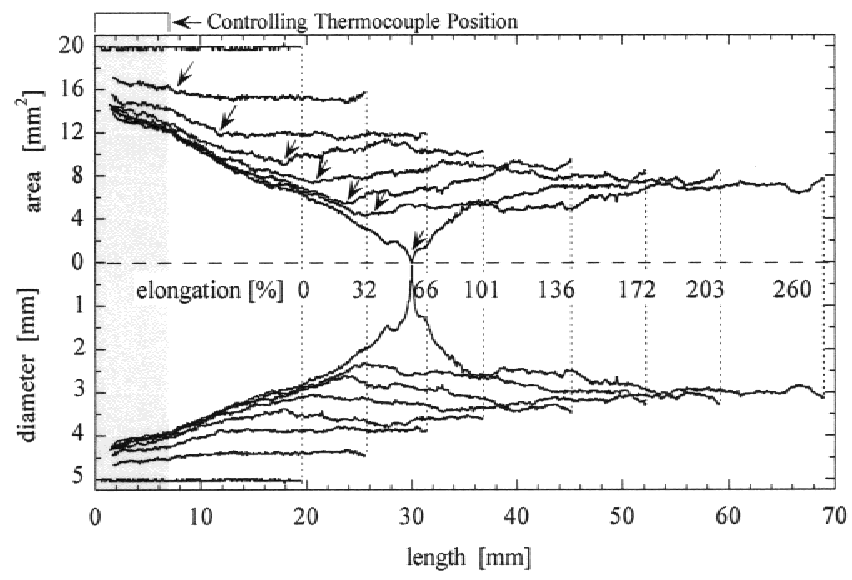

FIG. 5. Axial profiles of specimen diameter and cross-sectional area at several superplastic elongations, from the TSP thermal shielding experiment. Controlling thermocouple position is indicated by the shaded region, and the arrows follow the progression of a plastic instability. 


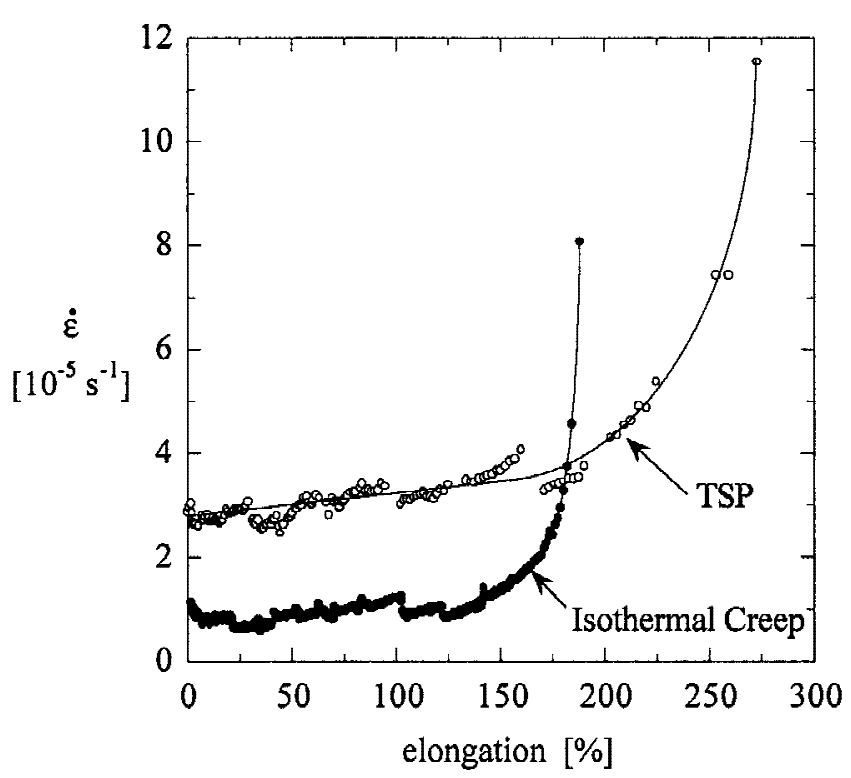

FIG. 6. Deformation histories of the unshielded creep and TSP fracture experiments, showing the strain rate $\dot{\epsilon}$ as a function of the elongation.

cycles. Second, the lower stress exponent of TSP (Fig. 2) gives rise to greater flow stability and thus results in larger strains to fracture. In Fig. 6, the elongation at failure during TSP at $4.5 \mathrm{MPa}$ is approximately $275 \%$, compared to approximately $180 \%$ for the isothermal creep experiment.

In the absence of thermal shielding, both creep and TSP deformation occurred symmetrically about the gauge midpoint, as observed in the specimen profiles in Fig. 7. However, the deformation was not uniform for either deformation mechanism, and failure was associated with a large, shallow neck near the gauge center for both creep and TSP deformation.

\section{DISCUSSION}

\section{A. Transformation superplasticity of Ti-6AI-4V}

Greenwood and Johnson ${ }^{18}$ developed a continuummechanics theory of TSP, in which an external uniaxial stress $\sigma$ biases internal strains produced by the transformation volume mismatch. For a material in which the weaker phase creeps with a power law, they derived a linear relationship between the strain increment produced on each thermal cycle, $\Delta \epsilon$, and the applied stress, $\sigma$ :

$$
\Delta \epsilon=\frac{4}{3} \cdot \frac{5 \cdot n}{4 \cdot n+1} \cdot \frac{\Delta V}{V} \cdot \frac{\sigma}{\sigma_{o}},
$$

where $n$ is the creep stress exponent of the weaker polymorph, $\Delta V / V$ is the transformation volume mismatch, and $\sigma_{o}$ is the average internal stress during the transformation, assumed to be much larger than $\sigma$.
The linear relationship of Eq. (1) has been observed by several authors ${ }^{9,10,17,19}$ for thermal cycling of Ti-6Al$4 \mathrm{~V}$. Although the value of the slope $\Delta \epsilon / \sigma$ is characteristic of the Ti-6Al-4V $\alpha-\beta$ transformation, it depends on the thermal cycle amplitude, because different amplitudes produce transformations of differing completeness (i.e., different effective volume changes, $\Delta V / V)$. The value observed at the beginning of TSP fracture experiments, $\Delta \epsilon / \sigma \approx 3.1 \mathrm{GPa}^{-1}$ (Figs. 4 and 6 ), is in agreement with our previous work ${ }^{9}$ using the same cycles $[\Delta \epsilon / \sigma \approx$ $3.1 \mathrm{GPa}^{-1}$, from the data in Fig. 2 fitted to Eq. (1)], as well as that of Kot et al., ${ }^{10}$ who found $\Delta \epsilon / \sigma \approx 3.2 \mathrm{GPa}^{-1}$ for thermal cycles in the range $760-980{ }^{\circ} \mathrm{C}$, at a fast rate of $\sim 30$ s per cycle. In Ref. 17, we reported a slope of $\Delta \epsilon / \sigma=2.1 \mathrm{GPa}^{-1}$, for 8 -min cycles between 840 and $990{ }^{\circ} \mathrm{C}$. The cycles in the present work involve a significant excursion into the $\beta$-field $\left(1000-1030^{\circ} \mathrm{C}\right)$ and thus contain an additional contribution to deformation from creep of the $\beta$-phase. The observed changes in $\Delta \epsilon / \sigma$ over the course of deformation (Figs. 4 and 6) are attributed to the development of plastic instabilities, as observed in Fig. 5, and are discussed in Sec. IV. C.

\section{B. Thermally initiated flow instabilities}

The importance of uniform thermal conditions for TSP flow stability is emphasized by the data in Fig. 4. When the specimen was slightly shielded from the radiant heaters by a thermocouple probe, the measured strain increments were about $25 \%$ lower than without shielding. Given a lower thermal flux, the specimen may not have completely transformed on heating, which would reduce the deformation expected from TSP. Furthermore, for the period of time during each cycle when the specimen is fully in the $\beta$-field $\left(>1000^{\circ} \mathrm{C}\right)$, power-law creep occurs instead of TSP. If the thermal shielding effect reduces the length of time spent in the $\beta$-field or the maximum temperature achieved during the cycling, the contribution of power-law creep in the $\beta$-phase is reduced.

The thermal effect described above was uniform over the gauge length of the specimen, and thus the deformation rate of the entire gauge section was affected. The implications of this experiment for nonuniform thermal conditions are clear; local hot or cold spots can experience substantially different deformation behavior that may lead to the nucleation and/or growth of plastic instabilities. For each stage of the shielded experiment, the controlling thermocouple was positioned on the specimen gauge section, about $7 \mathrm{~mm}$ from the upper specimen head (Fig. 5). This thermocouple thus provided partial thermal shielding for the upper $7 \mathrm{~mm}$ of the gauge, whereas the remainder of the specimen gauge was unshielded. As shown in Fig. 5, the position of the controlling thermocouple can be directly correlated with the onset of inhomogeneous deformation. The upper $7 \mathrm{~mm}$ of the specimen experienced only a small radial contraction, 
even after the specimen was deformed to $260 \%$ elongation. In contrast, sections of the gauge that were far from the controlling thermocouple deformed in a generally homogeneous manner. Furthermore, a diameter inhomogeneity that initiated near the thermocouple tip in the early stages of deformation spread and developed into a significant profile gradient over the course of superplastic elongation. The site of fracture seems to be associated with this inhomogeneity, as shown by the arrows in Fig. 5.

\section{Tensile creep and TSP fracture}

Barring environmental degradation or microstructural coarsening, the two most common modes of tensile creep fracture are by the accumulation of internal damage (cavity growth and coalescence) or external macroscopic damage (necking or loss of section). ${ }^{20}$ In the present case of TSP in coarse-grained Ti-6Al-4V, almost no cavitation damage was detected (Fig. 3), and all of the specimens were pulled to failure at a fine point. These results are similar to those for fine-grained Ti-6Al-4V deformed isothermally in the grain- or phase-boundary sliding regimen, in which superplastic flow without significant cavitation has been observed up to elongations $>1000 \% .^{21}$

It is apparent from Figs. 3 and 5 that macroscopic plastic instabilities govern the tensile ductility during TSP of Ti-6Al-4V and that cavitation plays a negligibly small role. Under these conditions, the expected ductility is primarily a function of the stress exponent of deformation, $n^{22-25}$ [although the strain-hardening exponent also has an effect (considerably smaller than that of the stress exponent ${ }^{22}$ ) on tensile ductility, we do not discuss it here because neither creep nor TSP of Ti-6Al-4V exhibit strain hardening at these temperatures (Fig. 5)]. Following Hart's ${ }^{23}$ stability analysis for a geometric defect, Nichols ${ }^{22}$ gives the following relation that predicts tensile elongation $e_{f}$ (expressed as engineering strain in $\%$ ) as a function of $n$, assuming an initial cross-sectional variation $\delta$ :

$$
e_{f}=\left(\delta^{-1 / n}-1\right) \cdot 100
$$

This relationship has been experimentally validated for creep of a wide range of metallic materials by Nichols. ${ }^{22}$

For TSP of Ti-6Al-4V at $\sigma_{n}=4.5 \mathrm{MPa}$, the stress exponent is $n \approx 1.2$ (Fig. 2), due to the superposition of a linear TSP component (Eq. 1) and a nonlinear creep component that accumulates at temperatures outside the phase transformation range during thermal cycling $\left(>1000^{\circ} \mathrm{C}\right.$ ). Isothermal creep at the effective temperature $T_{\text {eff }}=982{ }^{\circ} \mathrm{C}$ occurs with a stress exponent $n \approx 2.1$ (Fig. 2). With these values of $n$, and assuming an upperbound initial cross-sectional variation $\delta=0.01$, Eq. (2) predicts tensile elongations of $4500 \%$ and $800 \%$ for TSP and creep, respectively. These values can be compared to the measured engineering strains of $275 \%$ and $180 \%$; clearly, the ductility of Ti-6Al-4V in the present experiments is compromised by some source of plastic instability other than a geometric defect.

The thermal shielding experiment (Fig. 5) showed that, under radiant heating conditions, thermal variations can initiate plastic instabilities during thermal cycling. Therefore, we hypothesize that thermal variations are present both during isothermal creep and thermal cycling, and are responsible for the unexpectedly low tensile elongations in both creep and TSP (Figs. 6 and 7). Although there was no shadowing of the gauge section in the experiments of Figs. 6 and 7, the specimen heads were held by $\mathrm{Ni}$-base superalloy grips, which shielded them and caused the head temperature to be somewhat below that of the gauge section. There was, therefore, a thermal gradient in the specimens near the specimen heads. Such a gradient is evidenced by the profiles in Fig. 7, which show that gauge deformation was slower near the heads, compared with the central portion of the gauge. This is true for both isothermal creep, in which there was apparently a static thermal gradient, and for TSP, in which the thermal gradients were dynamic because of thermal cycling.

In the following sections, we implement a numerical model of creep and TSP tensile experiments, to examine the effects of thermal variations on the expected ductility.
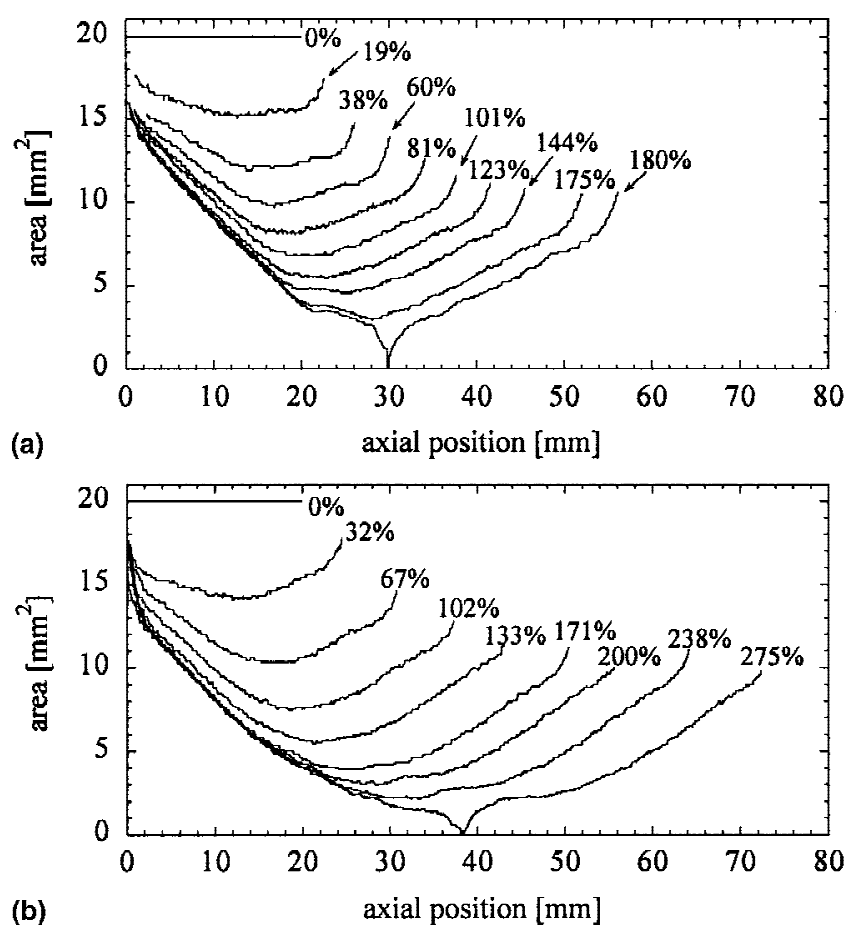

FIG. 7. Axial profiles of the tensile specimens during the unshielded tensile fracture experiments for several elongations; (a) isothermal creep and (b) transformation superplasticity. 
The purpose of this analysis is to establish the magnitude of the thermal effects in the present experiment, and thereby explain the cause of the unexpectedly low-tensile elongations (which are, however, high enough for most superplastic-forming applications). Implications for potential industrial TSP shape-forming operations are also briefly discussed.

\section{Numerical analysis of uniaxial creep}

The numerical model used here was developed by Burke and $\mathrm{Nix}^{24}$ as well as Semiatin and coworkers, ${ }^{26-28}$ who validated it against experiments on steel, two-dimensional finite-difference computations, and three-dimensional finite-element mechanics models. The model is one-dimensional, in that the tensile gauge section is divided into $\mathrm{M}$ slices perpendicular to the tension axis, and each slice is required to obey the axial force-balance equation:

$$
\sigma_{x}(x) \cdot A(x)=F_{A},
$$

where $\sigma_{x}$ is the average stress in the tension axis, $A$ is the cross-sectional area of the slice, $x$ is the axial position, and $F_{A}$ is the applied tensile force. As nonuniformities develop in the specimen profile, the stress state is no longer one of simple tension, but becomes triaxial. Bridgman $^{29}$ derived a correction factor that relates the effective axial stress, $\sigma_{e}$, to the average stress $\sigma_{x}$ :

$$
\sigma_{e}=\sigma_{x} \cdot\left[\left(1+\frac{2 \cdot R}{a}\right) \cdot \ln \left(1+\frac{a}{2 \cdot R}\right)\right]^{-1},
$$

where $R$ is the radius of curvature in the profile of the gauge section, and $a$ is the local diameter of the cylindrical tensile specimen. Finally, the effective axial stress is related to the strain rate through a typical creep power law:

$$
\dot{\epsilon}=K \cdot \exp \left(\frac{-Q}{R_{g} T}\right) \cdot \sigma_{e}^{n},
$$

where $K$ is a temperature-independent constant, $Q$ is the creep activation energy, $R_{g}$ is the gas constant, and $T$ is the absolute temperature.

The computations proceed as follows. For a given slice, the local profile radius of curvature is calculated from the diameters of the neighboring slices (formulas are available in Ref. 27). Knowing the applied force and the local diameter and radius of curvature, Eqs. (3) and (4) are used to calculate the effective axial stress. Eq. (5) is then used to compute the instantaneous strain rate in each slice. A finite time increment $\Delta t$ is applied, and the strain developed in each slice is found as $\dot{\epsilon} \cdot \Delta t$. The change in diameter (and cross-sectional area) are computed by assuming volume constancy in each slice.
Thus, the full specimen profile is known at each time step, and the elongation and average strain rate can be calculated in a straightforward manner.

To apply this model to the present isothermal creep experiment, we use $M=1000$ slices to represent onehalf of the gauge section; mirror symmetry is imposed at the gauge midpoint. Experimental values are taken for the apparent activation energy of deformation at $982{ }^{\circ} \mathrm{C}, Q=430 \mathrm{~kJ} / \mathrm{mol}$ (Fig. A1), the stress exponent $n=2.1$ (Fig. 2), and the creep constant $\mathrm{K}=5.5$. $10^{11} \mathrm{MPa}^{-2.1} \cdot \mathrm{s}^{-1}$ (Figs. 2 and A1). During the experiment, the applied nominal stress, $\sigma_{n}=4.5 \mathrm{MPa}$, was calculated based on the assumption of uniform gauge deformation. Therefore, the applied force depends on the instantaneous specimen gauge length, $L$ :

$$
F_{A}=\sigma_{n} \cdot \frac{A_{o} \cdot L_{o}}{L},
$$

where $A_{o}$ and $L_{o}$ are the initial cross-sectional area and length of the gauge.

Finally, we introduce a thermal gradient in the gauge section of the specimen. Following our earlier discussion, we take the ends of the gauge (near the specimen heads) to be at a temperature $\Delta T$ below the temperature at the center of the gauge, $T_{c}=982{ }^{\circ} \mathrm{C}$. Furthermore, as a first approximation, we assume that the thermal gradient is triangular, and described in the range $0 \leqslant$ $x \leqslant L / 2$ by:

$$
T=T_{c}-\Delta T \cdot\left(1-2 \cdot \frac{x}{L}\right),
$$

at all stages of the computation. This thermal profile is quite simple and probably not physically realistic. However, here we attempt only to show that thermal gradients compromise tensile ductility, and the simple triangular gradient is sufficient for this purpose. A more detailed analysis of nonisothermal effects, including conduction, convection in the test atmosphere, and mechanical heat generation, is described in Ref. 27, and is beyond the scope of the present work.

Figure 8 shows the experimental strain-rate history of the isothermal creep experiment $\left(T=982{ }^{\circ} \mathrm{C}, \sigma_{n}=\right.$ 4.5 MPa) from Fig. 6, compared with the output of the numerical model for several thermal inhomogeneities, $\Delta T$. The model results clearly show the effect of thermal gradients on tensile ductility; with a negligible thermal gradient $(\Delta T=0.1 \mathrm{~K})$, the model predicted a failure elongation of $>1000 \%$. In contrast, with a reasonable temperature variation of $\Delta T=12 \mathrm{~K}$, the numerical model closely matched the experimental data, with fracture occurring at $e_{f} \approx 180 \%$. This strong ductility dependence on thermal variations is due to the large apparent activation energy $Q=430 \mathrm{~kJ} / \mathrm{mol}$, which is associated with globularization of the colony-type microstructure of Fig. $1 .^{30}$ 
By using the best-fit value $\Delta T=12 \mathrm{~K}$ from Fig. 8 , the specimen profiles predicted by the model can be directly compared with the experimental profiles from Fig. 7. Such a comparison is shown in Fig. 9 for four representative profiles. Despite the simple temperature distribution assumed in the model, the agreement with the experimental profiles is reasonable. In particular, the model predicts the minimum cross section at each strain with accuracy. A better match between experiment and model, particularly near the specimen heads, could be achieved by using a more complex thermal profile.

To summarize this section, the numerical model of Semiatin et $_{\text {al. }}{ }^{26-28}$ can be used to predict both the strainrate history and the specimen profiles of $\mathrm{Ti}-6 \mathrm{Al}-4 \mathrm{~V}$ dur-

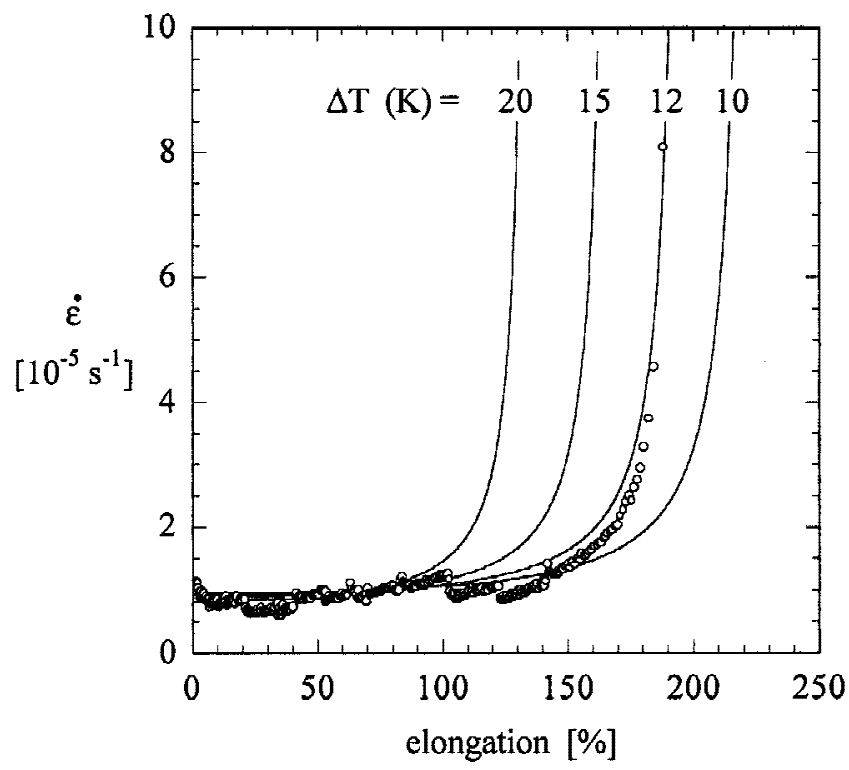

FIG. 8. Comparison of the experimental strain rate history (points) with the predictions of the numerical model (lines), during isothermal creep at $982{ }^{\circ} \mathrm{C}$ with $\sigma_{n}=4.5 \mathrm{MPa}$ and several thermal variations, $\Delta T$.

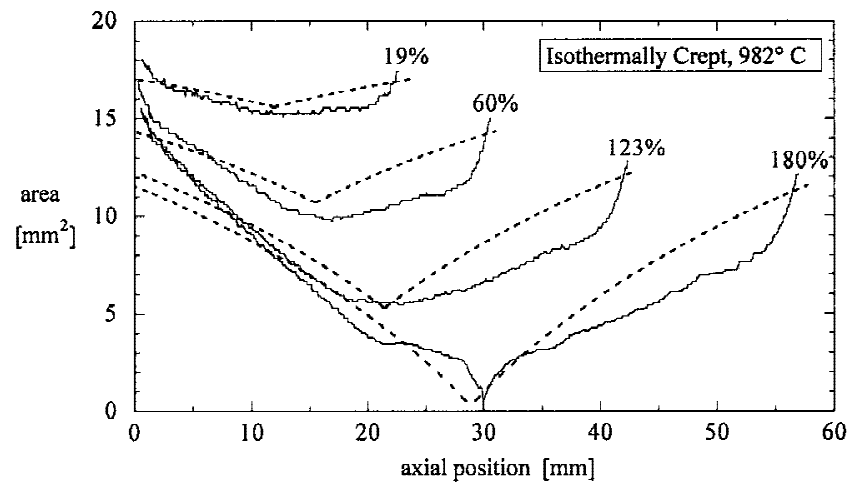

FIG. 9. Axial specimen profiles for isothermal creep at $982{ }^{\circ} \mathrm{C}$ for several gauge elongations. Experimental profiles (solid lines) are compared with the predictions of the numerical model (dashed lines) with thermal variation of $\Delta T=12 \mathrm{~K}$. ing isothermal creep at $982{ }^{\circ} \mathrm{C}$, using as a simple adjustable parameter a temperature variation of about $12 \mathrm{~K}$ between gauge center and head.

\section{Numerical analysis of transformation superplasticity}

Applying the above numerical model to TSP is considerably more complicated than for isothermal creep. To do so rigorously would require knowledge of the instantaneous strain rate at every moment during the thermal cycle. As discussed in Ref. 17, this is a complex problem, because TSP is dependent on the rate of phase transformation and the instantaneous fraction transformed, as well as temperature. Furthermore, the dynamic thermal situation during cycling may cause moving temperature gradients, making approximation of the instantaneous temperature at every position along the gauge quite difficult.

As a simple, first approximation to the complex deformation during thermal cycling, we model isothermal deformation at the average, or effective, temperature of the thermal cycles, $T_{\text {eff }}=982{ }^{\circ} \mathrm{C}$ (Appendix). In this case, deformation proceeds according to Eq. (5), where the strain rate is related to the strain increment developed after each cycle from Eq. (1) as $\dot{\epsilon}=\Delta \epsilon / \Delta t$, where $\Delta t$ is the cycle duration. On average, then, deformation occurs with a stress exponent of $n=1.2$ (Fig. 2), and we again assume a simple triangular effective temperature distribution of amplitude $\Delta T$.

It is difficult to assign a numerical value for the activation energy, $Q$, for TSP of Ti-6Al-4V. If one section of the gauge experiences a lower average temperature than another, it will cycle between different volume fractions of $\beta$-phase and will thus experience a different extent of phase transformation. In addition, the internal stress of the transformation is relaxed by creep of the weaker, $\beta$-phase, which obeys an Arrhenius temperature dependence itself. As an upper bound on the effect of $\Delta T$ on tensile ductility (or a lower bound on elongation to fracture), we take $Q=430 \mathrm{~kJ} / \mathrm{mol}$, the same as for isothermal creep (Appendix). This assumption will be discussed later in this section. With this value of $Q$, the parameter $K=1.9 \cdot 10^{15} \mathrm{MPa}^{-1.2} \cdot \mathrm{s}^{-1}$ is found by fitting the data in Fig. 2 to Eq. (5).

The strain-rate histories predicted by the numerical model are shown in Fig. 10 for TSP of Ti-6Al-4V. The best agreement with the experimental data is found with a thermal variation of $\Delta T=25 \mathrm{~K}$, for which the experimental elongation to fracture was about $e_{f} \approx 275 \%$. By using the same value of $\Delta T$, the specimen profiles predicted by the model are shown in Fig. 11, compared with select experimental profiles from Fig. 7. The model is reasonably accurate in predicting the strain-rate history and the shape of the gauge section. We note here that 
the upper-bound choice of activation energy suggests a reasonable thermal variation of $25 \mathrm{~K}$ during the TSP experiment. Using a lower value of $Q$ in the model results in greater flow stability; with a lower-bound value of $Q=153 \mathrm{~kJ} / \mathrm{mol}$ (as for isothermal creep of $\alpha$ - or $\beta$-phase titanium ${ }^{31}$ ) and $\Delta T=25 \mathrm{~K}$, the model predicts $e_{f} \approx 700 \%$. Alternatively, a thermal variation far in excess of $25 \mathrm{~K}(\Delta T \approx 70 \mathrm{~K})$ would be required to account for the low experimental ductility. The value $\Delta T=25 \mathrm{~K}$ is reasonable for the dynamic conditions of thermal cycling imposed in the TSP experiment, but, given the high thermal conductivity of $\mathrm{Ti}-6 \mathrm{Al}-4 \mathrm{~V}$ and the slow rate of thermal cycling, a value of $\Delta T=70 \mathrm{~K}$ seems less

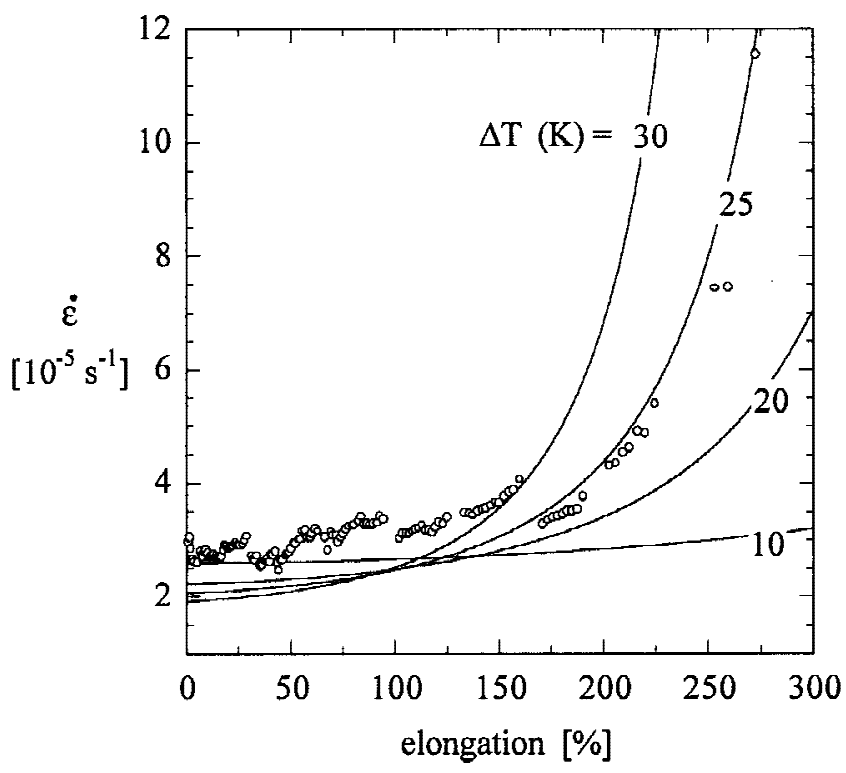

FIG. 10. Thermal cycling strain rate as a function of gauge elongation for the unshielded TSP experiment; experimental data (points) are compared with the numerical model predictions (lines) for several values of $\Delta T$.

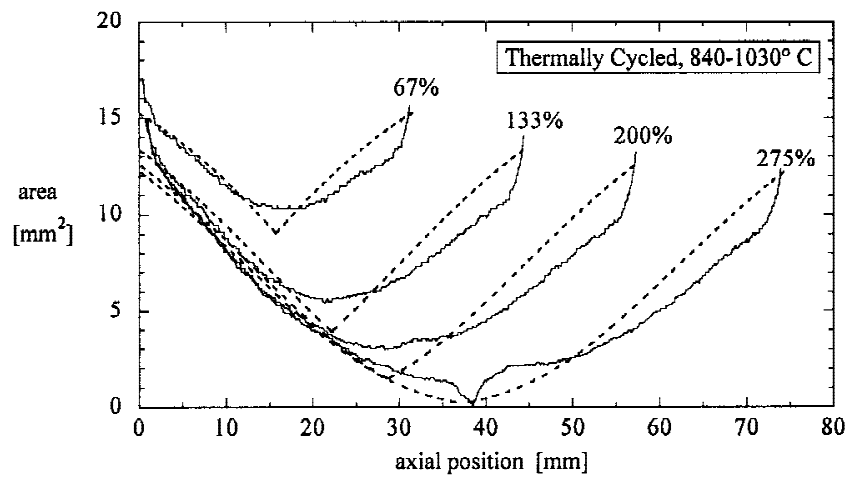

FIG. 11. Specimen profiles during transformation superplasticity at several elongations, comparing experimental data (solid) and model predictions (dashed). reasonable. In summary, the numerical model of TSP deformation requires the choice of two adjustable parameters (activation energy $Q$ and temperature variation $\Delta T$ ), both of which take reasonable values when the model is fitted to the experimental data.

The thermal variation $(\Delta T=25 \mathrm{~K})$ that best fitted the TSP data is about twice that required to fit the isothermal data $(\Delta T=12 \mathrm{~K})$. This result indicates that the TSP experiment has a larger degree of thermal variation, which is to be expected. This thermal variation drastically reduces the achievable tensile elongation (from an expected $4500 \%$ to only $275 \%$ ). However, inducing transformation superplasticity by thermal cycling offers the advantage of a lower stress exponent, which led to a substantial gain in tensile elongation over isothermal creep at the effective temperature, despite the larger thermal variation. For industrial implementation of TSP as a forming method, these issues may govern the selection of part size or thermal cycling rate (through the requirement of uniform dynamic thermal conditions) or may alternatively restrict the achievable forming ductility. Different heat sources (e.g., induction or direct Joule heating) may provide more stable dynamic thermal conditions than the radiant furnaces used here. In any case, without the development of internal cavitation damage, the formed parts should retain the mechanical properties of the bulk material, as also found in postdeformation mechanical testing. $6,9,10$

\section{CONCLUSIONS}

Failure of Ti-6Al-4V during tensile deformation by transformation superplasticity has been investigated during thermal cycling between 840 and $1030{ }^{\circ} \mathrm{C}$, through the $\alpha-\beta$ transformation range, under a uniaxial stress of 4.5 MPa. Isothermal creep fracture was examined at the same stress and at the average, effective temperature of the thermal cycles $\left(982^{\circ} \mathrm{C}\right)$. By periodically interrupting the straining with excursions to room temperature, internal cavity development and external plastic instabilities were assessed, leading to the following conclusions:

(1) Transformation superplasticity results in larger tensile elongation (275\%) than isothermal creep at the effective cycling temperature $(180 \%)$.

(2) Less than $0.4 \%$ cavities were detected in the Ti-6Al-4V specimens at any stage of deformation, for both isothermal creep and thermal cycling.

(3) Successive profiles of the specimen gauge sections illustrated the growth of macroscopic plastic instabilities (necks). Thermal variations have a significant impact on the deformation rate and are associated with the initiation of these inhomogeneities in the specimen cross section. A numerical model is used to show the effect of thermal gradients in reducing tensile ductility. 


\section{ACKNOWLEDGMENTS}

This study was primarily funded by National Science Foundation SBIRs, Grant Nos. 9760593 and 9901850, monitored by Dr. R. Coryell, through subcontracts from Dynamet Technology. Helpful discussions with W. Zimmer of Dynamet Technology are gratefully recognized, and C.S. acknowledges support of the United States Department of Defense through a National Defense Science and Engineering Graduate Fellowship.

\section{REFERENCES}

1. T.G. Nieh, J. Wadsworth, and O.D. Sherby, Superplasticity in Metals and Ceramics. (Cambridge University Press, Cambridge, 1997).

2. C. Schuh and D.C. Dunand, Acta Mater. 46, 5663 (1998).

3. P. Zwigl and D.C. Dunand, Mater. Sci. Eng. (in print).

4. D.C. Dunand and C.M. Bedell, Acta Mater. 44, 1063 (1996).

5. P. Zwigl and D.C. Dunand, Metall. Mater. Trans. 29A, 565 (1998).

6. C. Schuh, W. Zimmer, and D.C. Dunand, in Creep Behavior of Advanced Materials for the 21st Century, edited by R.S. Mishra, A.K. Mukherjee, and K.L. Murty (TMS, Warrendale PA, 1999), p. 61.

7. C. Schuh and D.C. Dunand, Scripta Mater. 40, 1305 (1999).

8. D.C. Dunand and S. Myojin, Mater. Sci. Eng. 230A, 25 (1997).

9. C. Schuh and D.C. Dunand, Int. J. Plastic. (in print).

10. R. Kot, G. Krause, and V. Weiss, in The Science, Technology and Applications of Titanium, edited by R.I. Jaffe and N.E. Promisel (Pergamon, Oxford, 1970), p. 597.

11. G. Gonzalez-Doncel and O.D. Sherby, Metall. Mater. Trans. 27A, 2837 (1996).

12. S. Abkowitz, P.F. Weihrauch, and S.M. Abkowitz, Industrial Heating 12, 32 (1993).

13. P. Zwigl and D.C. Dunand, Metall. Mater. Trans. 29A, 2571 (1998).

14. D.W. Green and J.O. Maloney, eds. Perry's Chemical Engineer's Handbook (McGraw-Hill, New York, 1984).

15. W. Szkliniarz and G. Smolka, J. Mater. Proc. Tech. 53, 413 (1995).

16. E. Sato and K. Kuribayashi, Acta. Metall. Mater. 41, 1759 (1993).

17. C. Schuh and D.C. Dunand, Acta Mater. (in print).

18. G.W. Greenwood and R.H. Johnson, Proc. Roy. Soc. Lond. 283A, 403 (1965).

19. K. Sato, T. Nishimura, and Y. Kimura, Mater. Sci. Forum 170-172, 207 (1994).

20. M.F. Ashby and B.F. Dyson, in Advances in Fracture Research, Fracture 84, edited by S.R. Valluri, et al. (Pergamon, Oxford, 1984), p. 3.

21. M.T. Cope and N. Ridley, Mater. Sci. Technol. 2, 140 (1986).

22. F.A. Nichols, Acta Metall. 28, 663 (1980).

23. E.W. Hart, Acta Metall. 15, 351 (1967).

24. M.A. Burke and W.D. Nix, Acta Metall. 23, 793 (1975).

25. A.K. Ghosh and R.A. Ayres, Metall. Trans. 7A, 1589 (1976).

26. S.L. Semiatin, A.K. Ghosh, and J.J. Jonas, Metall. Trans. 16A, 2291 (1985).

27. S.L. Semiatin, R.A. Ayres, and J.J. Jonas, Metall. Trans. 16A, 2299 (1985).

28. C.M. Lombard, R.L. Goetz, and S.L. Semiatin, Metall. Trans. 24A, 2039 (1993).

29. P.W. Bridgman, Trans. ASM 32, 553 (1944).

30. T. Seshacharyulu, S.C. Medeiros, J.T. Morgan, J.C. Malas, W.G. Frazier, and Y.V.R.K. Prasad, Scripta Mater. 41, 283 (1999).
31. H.J. Frost and M.F. Ashby, Deformation-Mechanism Maps: The Plasticity and Creep of Metals and Ceramics (Pergamon Press, Oxford, 1982).

32. T. Seshacharyulu, S.C. Medeiros, J.T. Morgan, J.C. Malas, W.G. Frazier, and Y.V.R.K. Prasad, Mater. Sci. Eng. A 279, 289 (2000).

\section{APPENDIX}

\section{Effective temperature during thermal cycling}

The effective temperature of a thermal cycle is the temperature at which the isothermal creep rate $\dot{\epsilon}_{i}$ is equal to the average rate during thermal cycling, if there were no enhancement due to the phase transformation. Mathematically, it is the temperature that satisfies the following condition:

$$
\dot{\epsilon}_{i}=\frac{1}{\Delta t} \int_{\Delta t} \dot{\epsilon}[T(t)] \cdot d t
$$

where $\Delta t$ is the thermal cycle duration. For many practical cases, including transformation superplasticity of allotropic metals (which transform at a single temperature $)^{4}$ and thermal cycling creep of metals and composites, ${ }^{16}$ this amounts to averaging the Arrhenius term of the creep law [Eq. (5)], $\exp \left(-Q / R_{g} T\right)$, over the cycle temperatures. For thermal cycles through the $\alpha+\beta$ range of $\mathrm{Ti}-6 \mathrm{Al}-4 \mathrm{~V}$, the instantaneous creep rate is related to temperature through the relative volume fractions of the two phases, as well as the creep laws of those phases and the micromechanisms of their compatible deformation. ${ }^{30,32}$ Rather than model this complex

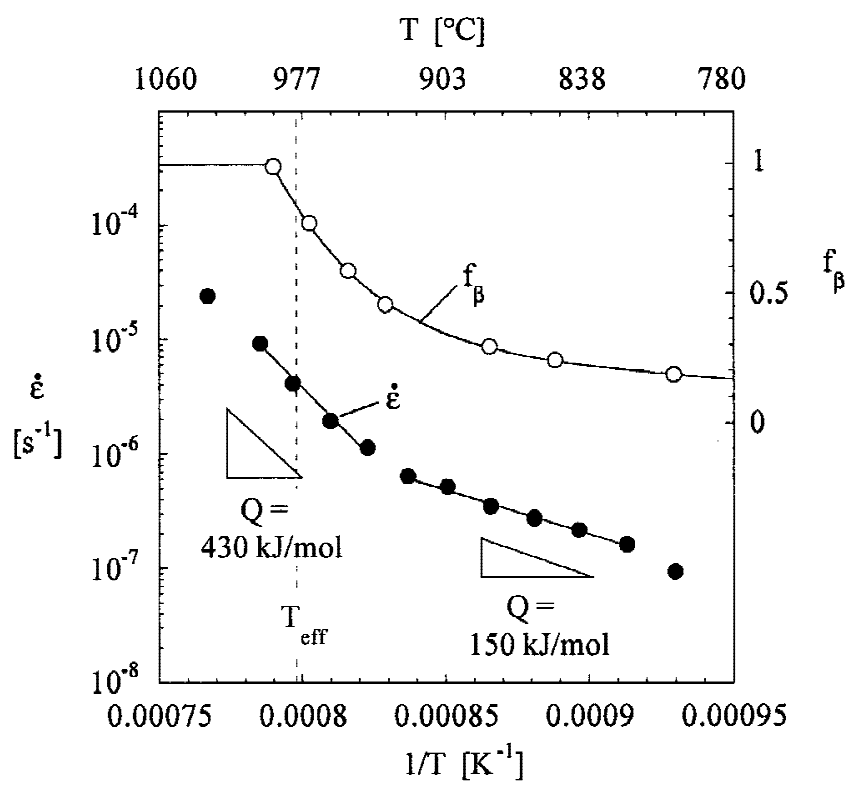

FIG. A1. Isothermal creep rate of Ti-6Al-4V at various temperatures in the two-phase $\alpha+\beta$ field, with applied uniaxial stress of $4.5 \mathrm{MPa}$. Point at $1030{ }^{\circ} \mathrm{C}$ is taken from Ref. 17 . Volume fraction of $\beta$-phase, $f_{\beta}$, is from Ref. 15. 
temperature dependence, we have performed isothermal creep experiments at several temperatures in the $\alpha+$ $\beta$ range, as described below.

Isothermal creep experiments were conducted on rolled Ti-6Al-4V from Titanium Industries (Wooddale, IL), with the same specimen geometry and in the same creep apparatus described in the text. The applied stress was $\sigma=4.0 \mathrm{MPa}$, and the test temperatures were between 800 and $1030{ }^{\circ} \mathrm{C}$. At each temperature, sufficient time was allowed to develop a steady-state creep rate, after which the temperature was raised to the next value. The steady-state creep rates are shown as a function of the reciprocal temperature in Fig. A1, which illustrates two regions with activation energies $Q=430 \mathrm{~kJ} / \mathrm{mol}$ $\left(T>920{ }^{\circ} \mathrm{C}\right)$ and $Q=150 \mathrm{~kJ} / \mathrm{mol}\left(T<920^{\circ} \mathrm{C}\right)$. The acti- vation energy of the high-temperature region is consistent with data of Ref. $30(Q \approx 453 \mathrm{~kJ} / \mathrm{mol})$ at similar temperatures and higher strain rates $(\dot{\epsilon}=0.001-$ $\left.100 \mathrm{~s}^{-1}\right)$. The activation energy at lower temperatures $(Q \approx 150 \mathrm{~kJ} / \mathrm{mol})$ is consistent with self-diffusion of $\alpha-\mathrm{Ti}^{31}$ and is correlated to the large volume fraction of $\alpha$-phase at these temperatures $\left(60-80 \%,{ }^{15} \mathrm{Fig}\right.$. A1).

The effective temperature is found by fitting the experimental data of Fig. A1 with a smooth polynomial function and numerically evaluating Eq. (A1) for the thermal cycles used in the TSP experiments (840$\left.1030{ }^{\circ} \mathrm{C}\right)$. The resulting effective temperature is found to be $T_{\text {eff }}=982{ }^{\circ} \mathrm{C}$. Near $T_{\text {eff }}$, the creep rate is reasonably described by an Arrhenius temperature dependence, with an apparent activation energy $Q=430 \mathrm{~kJ} / \mathrm{mol}$. 